de la mise bas, alors que la capacité d'ingestion est limitée chez les femelles légères. Ceci entraîne un déficit alimentaire en début de lactation, déficit qui est aggravé par une portée nombreuse et par le début d'une nouvelle gestation. Pour faire face à la diminution du poids des reproducteurs, un nouveau critère de sélection, le poids du lapereau à l'âge de 63 jours, a été ajouté à l'objectif de sélection de la souche A1077. Cette souche est diffusée; il est donc malaisé d'analyser les conséquences de cette modification de l'objectif sur la fécondité et ses composantes, sur les qualités bouchères et sur l'efficacité alimentaire. Deux expériences de sélection permettront d'analyser cette question et d'entreprendre une étude génétique et biologique des caractéristiques de la croissance ; deux souches de formats initiaux différents seront soumises à une sélection divergente sur le poids à l'âge de 63 jours. Après cinq générations de sélection les évolutions de la fécondité, de l'efficacité alimentaire, des qualités bouchères et des qualités de la viande seront analysées.

\section{Références}

Bolet G., Esparbie J., Falières J., 1996. Relations entre le nombre de fœetus par corne utérine, la taille de portée à la naissance et la croissance pondérale des lapereaux. Ann. Zootech., 45, 185-200.

Castellini C., 1996. Recent advances in rabbit artificial insemination. 6e Congrès mondial de Cuniculture, Toulouse, 9-12 juillet 1996, 2, 13-28.
Fortun-Lamothe L., 1998. Effets de la lactation, du bilan énergétique et du rythme de reproduction sur les performances de reproduction chez la lapine primipare. $7^{\text {e }}$ Journées de la Recherche Cunicole en France, Lyon, 13-14 mai 1998. INRA-ITAVI, Paris, 257-260.

Fortun-Lamothe L, Bolet G., 1995. Les effets de la lactation sur les performances de reproduction chez la lapine. INRA Prod. Anim., 8, 49-56.

Fortun-Lamothe L., Bolet G., 1998. Relations entre le format, l'évolution des réserves corporelles et les performances de reproduction chez la lapine primipare : comparaison de deux types génétiques. 7 es Journées de la Recherche Cunicole en France, Lyon, 13-14 mai 1998. INRA-ITAVI, Paris, 27-30.

Kœhl P.F., 1997. GTE RENALAP 96 : une lapine produit $118 \mathrm{~kg}$ de viande par an. Cuniculture, 138, 247252

Rinaldo D., Bolet G., 1988. Effect of selection for litter size at weaning on reproductive life of female rabbits. $4^{\mathrm{e}}$ Congrès mondial de Cuniculture, Budapest, 10-14 octobre 1988, Genetics and Physiology, 269-275.

Rochambeau H. de, 1998. La femelle parentale issue des souches expérimentales de l'INRA : évolutions génétiques et perspectives. 7 es Journées de la Recherche Cunicole en France, Lyon, 13-14 mai 1998. INRA-ITAVI, Paris, 3-14.

Theau-Clément M., Roustan A., 1992. A study on relationships between receptivity and lactation in the doe and their influence on reproductive performance. J. Appl. Rabbit Res., 15, 412-421.

Tudela F., Poujardieu B., Gauzère J.M., 1998. Productivité de la lapine : préparation des reproducteurs. $7^{\text {es }}$ Journées de la Recherche Cunicole en France, Lyon, 13-14 mai 1998. INRA-ITAVI, Paris, 269-271.

\section{J.-P. BIDANEL}

INRA Station

de Génétique

quantitative

et appliquée

78352 Jouy-en-Josas

Cedex

\title{
Conséquences génétiques de l'accroissement de la prolificité des truies
}

La généralisation des programmes de sélection " hyperprolifiques » a conduit ces dernières années à une amélioration substantielle de la taille de la portée à la naissance dans les élevages de sélection et de multiplication. Les effets de cette sélection sont désormais perceptibles au niveau des élevages de production. Cet accroissement de la prolificité des truies va vraisemblablement s'amplifier dans les années à venir, lorsque les effets liés à la mise en place d'une évaluation génétique de type BLUP-modèle animal et à l'introduction des lignées synthétiques sino-européennes ou des races chinoises dans les schémas de croisement se feront sentir. Ce texte fait le point sur les conséquences génétiques de cette augmentation de la taille de la portée à la naissance à partir des résultats expérimentaux et des estimations de paramètres génétiques disponibles dans la littérature.

\section{1 / Conséquences génétiques d'une sélection sur la prolificité}

\section{1 / Sur les performances de reproduction}

La sélection pour la prolificité est le plus souvent basée sur le nombre de porcelets nés totaux (NT) par portée. Ce choix n'est pas 
sans conséquence. Il conduit certes à un accroissement des nombres de porcelets nés vivants $(\mathrm{NV})$ et sevrés (SEV) par portée, mais également à une augmentation sensible, plus que proportionnelle, du nombre de porcelets mort-nés (tableau 1). Cet accroissement du nombre de mort-nés pourrait en partie être dû à une augmentation de la durée de la mise bas liée à une moindre aptitude des utérus de grande taille à éjecter les porcelets, ainsi qu'aux poids plus faibles et plus hétérogènes des porcelets issus de portées de grande taille.

Les variations du taux de survie entre la naissance et le sevrage (TS) sont plus difficiles à appréhender du fait de la fréquence des échanges de porcelets entre portées. Les estimations de corrélations génétiques entre TS et NT ou NV sont dans l'ensemble faibles, avec une valeur moyenne légèrement négative pour NT et positive pour NV (tableau 2). Une sélection sur NT conduit également à une augmentation du poids total de la portée à la naissance et à 21 jours (tableau 2 ), ce dernier traduisant un accroissement de la production laitière (PL) des truies. L'évolution favorable de PL est vraisemblablement liée pour l'essentiel à celle du nombre de porcelets, l'évolution de PL à nombre de porcelets constant n'étant pas connue. L'accroissement de NT aura par contre peu d'effets sur le nombre de tétines (NTET), les estimations de corrélations génétiques entre NTET et la taille de portée étant dans l'ensemble proches de zéro. On peut également noter que, si une sélection sur le taux d'ovulation (TO) a des effets limités sur NT, une sélection sur NT se traduit par un accroissement au moins équivalent de TO (Després et al 1992).

Les valeurs des corrélations génétiques entre les caractères de taille de portée et les autres caractères de reproduction sont en général faibles. Ainsi, les moyennes de la littérature pour les corrélations génétiques entre NT, NV ou SEV et l'âge à la puberté sont proches de zéro (tableau 2). Néanmoins, Després et al (1992) ont montré des différences notables de précocité sexuelle et de taux d'ovulation entre des truies de la lignée hyperprolifique Large White (LW) et des truies LW témoins. Les estimations de corrélations génétiques entre la prolificité des truies et les caractères sexuels mâles (croissance testiculaire, production spermatique) sont également en général proches de zéro (Rothschild et Bidanel 1997). Un certain antagonisme génétique entre l'intervalle sevrage ostrus (ISO) et la prolificité est rapporté dans une expérience hollandaise de sélection visant à réduire l'intervalle sevrage - saillie fécondante (ISSF) (Ten Napel, communication personnelle), mais aucune différence d'ISSF n'est observée par Després et al (1992) entre truies LW hyperprolifiques et truies témoins.

\section{2 / Sur les performances de production}

L'augmentation de la taille de la portée à la naissance se traduit par une diminution du
Tableau 1. Réponses à une sélection sur la prolificité dans 5 expériences de sélection (d'après Blasco et al 1995).

\begin{tabular}{|l|c|c|c|}
\hline \multirow{2}{*}{\multicolumn{1}{|c|}{ Référence }} & \multicolumn{3}{|c|}{ Caractère } \\
\cline { 2 - 4 } & Nés totaux & Nés vivants & Mort-nés \\
\hline Petit et al 1988 & 1,0 & 0,8 & 0,2 \\
Herment et al 1994 & 1,3 & 1,0 & 0,3 \\
Casey et al 1994 & 1,7 & 1,2 & 0,5 \\
Bolet et al 1998 & 2,2 & 1,2 & 1,0 \\
Sorensen (non publié) & 0,8 & 0,4 & 0,4 \\
\hline
\end{tabular}

Tableau 2. Corrélations génétiques entre la taille de portée et d'autres caractères de reproduction (d'après Rothschild et Bidanel 1997).

\begin{tabular}{|l|c|c|c|}
\hline & Nés totaux & Nés vivants & Sevrés \\
\hline Age à la puberté & 0,04 & 0,04 & 0,10 \\
Taux d'ovulation & 0,16 & 0,16 & 0,10 \\
Taux de survie naissance-sevrage & $-0,11$ & 0,16 & 0,53 \\
Poids de la portée & & & \\
$\quad$ - à la naissance & 0,62 & 0,65 & 0,70 \\
- à 21 jours & 0,45 & 0,61 & 0,80 \\
\hline
\end{tabular}

poids moyen des porcelets à la naissance et de leur croissance pendant la période d'allaitement. Cet effet peut avoir des conséquences jusqu'en fin d'engraissement, comme tendent à l'indiquer les corrélations génétiques défavorables entre la taille de la portée et l'âge à $100 \mathrm{~kg}$ récemment obtenus en race Large White par Ducos et Bidanel (1996) ou Maignel et al (1998). Après le sevrage, les estimations des corrélations génétiques entre la prolificité et la vitesse de croissance sont par contre proches de zéro. Les liaisons génétiques entre la taille de la portée et l'épaisseur de lard dorsal ou la teneur en muscle de la carcasse sont également faibles, mais le plus souvent légèrement défavorables. Un certain antagonisme génétique pourrait par contre exister entre la consommation alimentaire en période de croissance et la prolificité des truies, comme tendent à le suggérer certains résultats récents (tableau 3 ).

Les liaisons génétiques entre les caractères de reproduction et de qualité de la viande sont quant à elles mal connues, mais vraisembla-

Tableau 3. Effets d'une réduction de l'appétit en période de croissance sur les performances de la truie (d'après Kerr et Cameron 1996). CMJ+, CMJ- = lignées sélectionnées pour, respectivement, une augmentation et une diminution de la consommation moyenne journalière.

\begin{tabular}{|l|c|c|c|}
\hline & Lignée CMJ- & Lignée CMJ+ & Signification \\
\hline $\begin{array}{l}\text { Epaisseur de lard dorsal à la mise bas } \\
\text { Consommation alimentaire }\end{array}$ & 25,7 & 30,7 & $*$ \\
pendant la lactation & 129 & 146 & $* *$ \\
Nés vivants & 11,2 & 10,7 & \\
Gain moyen quotidien des porcelets & & & $*$ \\
avant sevrage & 167 & 195 & $*$ \\
Poids de la portée à la naissance & 13,7 & 12,7 & $*$ \\
Poids de la portée au sevrage & 64,0 & 74,4 & $*$ \\
\hline
\end{tabular}

$*=\mathrm{P}<0,05 ; * *=\mathrm{P}<0,01$. 
blement assez proches de zéro. Hermesch et al (1995) présentent des corrélations génétiques non significatives entre la taille de la portée et le $\mathrm{pH}$ ou la perte au ressuyage. De même, Larzul (1997) n'observe aucune évolution notable de la prolificité dans une expérience de sélection visant à réduire le potentiel glycolytique du muscle. Des corrélations négatives entre la taille de la portée à la naissance et la couleur de la viande sont toutefois rapportées par Hermesch et al (1995).

Il convient également de noter que l'accroissement de la pression de sélection sur la prolificité se traduit inéluctablement par une baisse de la pression de sélection, et donc un progrès génétique moins important, pour les autres caractères de l'objectif de sélection. Ce phénomène est particulièrement sensible chez les verrats des lignées hyperprolifiques, qui sont en outre pénalisés par l'âge souvent relativement élevé de leur mère.

\section{2 / Conséquences liées à l'utilisation des races prolifiques chinoises}

\section{1 / Sur les performances de reproduction}

L'utilisation en croisement des races prolifiques chinoises a quant à elle des effets favorables, non seulement sur la taille de la portée à la naissance, mais également sur la plupart des autres caractères de reproduction. Comme l'ont montré Petit et al (1988), ces effets varient toutefois de façon importante en fonction de l'élevage. Les races Meishan et Jiaxing présentent, aussi bien en race pure qu'en croisement, des aptitudes maternelles nettement supérieures à celle des races européennes ou nord-américaines, qui se traduisent, à taille de portée équivalente, par des taux de survie entre la naissance et le sevrage nettement supérieurs (Bidanel 1993, Lee et Haley 1995). Cette supériorité est en partie liée au nombre plus élevé de tétines, mais également aux caractéristiques comportementales des truies pures ou croisées Meishan. Schouten et Meunier-Salaun (1990) ont ainsi observé que les femelles Meishan avaient davantage de contacts avec leurs porcelets et allaitaient plus longtemps que des truies européennes. La production laitière des truies Meishan n'apparaît en revanche pas supérieure à celle de femelles européennes (Van der Steen et de Groot 1992), contrairement à celle des truies F1 Meishan x Large White, nettement plus élevée. Enfin, les truies Meishan présentent également des taux de mise bas plus élevés et des intervalles sevrage - œstrus réduits par rapport aux races occidentales (Després et al 1992). Ces bonnes caractéristiques de reproduction se retrouvent en partie dans les lignées sino-européennes, même si aucune comparaison rigoureuse avec les populations maternelles actuellement utilisées en Europe n'a pour l'instant été réalisée.

\section{2 / Sur les performances de production}

L'utilisation en croisement des races chinoises conduit à une détérioration des performances de production des produits terminaux, en particulier de carcasse (rendement, teneur en viande maigre) par rapport aux plans de croisement utilisant des types génétiques classiques (Legault et al 1985). Cette détérioration est proportionnelle au pourcentage de gènes Meishan chez les produits terminaux (Bidanel et al 1993), mais son impact économique est variable du fait notamment de la non linéarité de la liaison entre le prix de vente et le classement commercial des carcasses. Les effets sur la croissance et l'efficacité alimentaires sont beaucoup plus réduits du fait de l'importance des effets d'hétérosis sur ces caractères (Bidanel et al 1990 et 1993). Enfin, les porcs croisés Meishan tendent à avoir une meilleure qualité de viande que types génétiques européens (Bidanel et al 1993). La diminution des performances de production est moindre chez les produits terminaux issus de plans de croisement utilisant des lignées sino-européennes compte tenu de la sélection pour les performances de croissance et de carcasse pratiquée dans ces lignées. Cette diminution et, par voie de conséquence, l'intérêt économique des lignées sino-européennes, est directement liée à l'efficacité de la sélection pratiquée (Bidanel 1989).

\section{Références}

Bidanel J.P., 1989. Etude de stratégies de valorisation en croisement de la race Meishan. 3 - Evaluation comparée de différents systèmes de croisement. Journées Rech. Porcine en France, 21, 361-366.

Bidanel J.P., 1993. Estimation of crossbreeding parameters between Large White and Meishan porcine breeds. III. Dominance and epistatic components of heterosis on reproductive traits. Genet. Sel. Evol. $25,263-281$.

Bidanel J.P., Caritez J.C., Legault C., 1990. Estimation of crossbreeding parameters between Large hite and Meishan porcine breeds. II. Growth before weaning and growth of females during the growing and reproductive periods. Genet. Sel. Evol., 22, 431-445.

Bidanel J.P., Caritez J.C., Gruand J., Legault C. 1993. Growth, carcass and meat quality performance of crossbred pigs with graded proportions of Meishan genes. Genet. Sel. Evol., 25, 83-99.

Blasco A., Bidanel J.P., Haley C.S., 1995. Genetics and neonatal survival. In : M.A. Varley (ed), The neonatal pig. Development and survival, 17-38. CAB International, UK.

Bolet G., Bidanel J.P., Ollivier L., 1998. Selection for litter size in pigs : efficiency of closed and open nucleus strategies. Genet. Sel. Evol. (soumis pour publication).

Casey D., Rathje T.A., Johnson R.K., 1994. Second thoughts on selection for components of reproduction in swine. In : Proc. 5th World Congress on Genetics Applied to Livestock Production, 17, 315-318. 
Després P., Martinat-Botté F., Lagant H., Terqui M., Legault C., 1992. Comparaison des performances de reproduction des truies appartenant à trois génotypes : Large White(LW), Large White " Hyperprolifique "(LWH), Meishan (MS). Journées Rech. Porcine en France, 24, 25-30.

Ducos A., Bidanel J.P., 1996. Genetic correlations between production and reproductive traits measured on-farm, in the Large-White and French Landrace breeds. J. Anim. Breed. Genet., 113, 493-504.

Herment A., Runavot J.P., Bidanel J.P., 1994. Une nouvelle évaluation de l'intérêt de la voie hyperprolifique chez le porc. Journées Rech. Porcine en France, 315-320.

Hermesch S., Luxford B.G., Graser H.U., 1995. Genetic relationships between litter size and meat quality traits in Australian pigs. In : J.A.M. Van Arendonk (ed), Book of Abstracts of the European Association for Animal Production, G1.18.

Kerr J.C., Cameron N.D., 1996. Genetic and phenotypic relationships between performance test and reproduction traits in Large White pigs. Anim. Sci., $62,531-540$.

Larzul, C., 1997. Variabilité génétique d'une mesure in vivo du potentiel glycolytique musculaire chez le porc. Relations avec les performances, les caractéristiques du muscle et la qualité technologique des viandes. Thèse de Doctorat, Institut National Agronomique Paris-Grignon, France.
Lee G.J., Haley C.S., 1995. Comparative farrowing to weaning performance in Meishan and Large White pigs and their crosses. Anim. Sci., 60, 269-280.

Maignel L., Bidanel J.P., Guéblez R., 1998. Intérêt d'une pesée au sevrage dans le contrôle de performances en ferme. Journées Rech. Porcine en France, $30,101-107$.

Petit G., Runavot J.P., Legault C., Gruand J., 1988. Evaluation en race pure et en croisement de la lignée hyperprolifique Large White dans un réseau d'élevages de multiplication et de production. Journées Rech. Porcine en France, 20, 309-314.

Rothschild M.F., Bidanel J.P., 1997. Biology and genetics of reproduction. In : M.F. Rothschild and A. Ruvinsky (eds), The genetics of the pig, 313-343. $\mathrm{CAB}$ International, UK.

Shouten W.G.P., Meunier-Salaun M.C., 1990. Behavioural and physiological changes around farrowing in Meishan, Large White and crossbred Dutch Landrace x Great Yorkshire sows. In : M. Molénat and C. Legault (eds), Chinese pig symposium, July 5-6, 1990, Toulouse, France, 167-178.

Van der Steen H.A.M., de Groot P.N., 1992. Direct and maternal breed effects on growth and milk intake of piglets : Meishan versus Dutch breeds. Livest. Prod. Sci., 30, 361-374.

\section{Influence de la nutrition sur le fonctionnement de l'axe gonadotrope}

L'augmentation de la taille de la portée s'accompagne d'une élévation de la production laitière et de l'appétit des truies. Cependant, l'augmentation de l'appétit est généralement insuffisante pour couvrir les dépenses nutritionnelles associées au surcroît de production laitière. Aussi, le risque de déficit nutritionnel en lactation augmente-t-il avec la prolificité des truies. Ce déficit est susceptible d'altérer les performances ultérieures de reproduction des truies (revues : Aherne et Kirkwood 1985, Dourmad et al 1994, Quesnel et Prunier 1995a). Ainsi, chez la truie en première lactation, un déficit nutritionnel élevé induit un allongement de l'intervalle sevrage-ovulation et peut conduire à un abaissement du taux d'ovulation et de la survie embryonnaire (Zak et al 1997). Chez la truie multipare, les effets du niveau alimentaire pendant la lactation sur le retour en œstrus, la fécondité, la survie embryonnaire et la taille de la portée à la naissance sont très peu marqués (Kirkwood et al 1988a, Varley et Prime 1993). Les effets du déficit nutritionnel pendant la lactation sur la fonction de reproduction de la truie se manifestent donc avec le plus d'acuité chez la jeune femelle. Ce texte analyse les effets de la nutrition sur le moment et le taux d'ovulation après avoir fait un rappel sur la physiologie de l'axe hypothalamus-hypophyse-ovaires.

\section{1 / Principales caractéristiques du fonctionnement de l'axe gonadotrope}

Chez la truie mature, quel que soit le stade physiologique, des follicules sortent continuellement de la réserve des follicules primordiaux, entrent en croissance et dégénèrent pour la plupart (= atrésie). Cette phase de croissance des follicules jusqu'à $2 \mathrm{~mm}$ de diamètre est quasi indépendante des sécrétions des hormones gonadotropes LH et FSH (Driancourt et al 1995). Cinq à dix jours avant l'ovulation, une cinquantaine de follicules en croissance (de 2 à $5 \mathrm{~mm}$ ) sont recrutés sous l'influence d'un signal encore mal déterminé (équilibre entre gonadotropines, facteurs locaux ?). Parmi ces follicules, dix à trente
A. PRUNIER, H. QUESNEL

INRA Station de Recherches Porcines, 35590 Saint-Gilles 